\title{
Managing congenitally missing lateral incisors with single tooth implants
}

\author{
Abu-Hussein Muhamad ${ }^{1 *}$, Watted Nezar ${ }^{2}$ and Abdulgani Azzaldeen ${ }^{3}$ \\ ${ }^{1}$ University of Naples Federic II, Naples, Italy, Department of Pediatric Dentistry, University of Athens, Athens, Greece \\ ${ }^{2}$ Department of Orthodontics, Arab American University, Jenin, Palestine \\ ${ }^{3}$ Department of Conservative Dentistry, Al-Quds University, Jerusalem, Palestine
}

\begin{abstract}
Congenitally missing teeth are frequently presented to the dentist. Interdisciplinary approach may be needed for the proper treatment plan. Several treatment options exist for the replacement of congenitally missing lateral incisors. These options include canine substitution, resin bonded fixed partial dentures, cantilevered fixed partial dentures, conventional fixed partial dentures and single tooth implants. Depending on which treatment option is chosen, a specific criterion has to be addressed. Interdisciplinary treatment plays a vital role to achieve an excellent, esthetic result for a most predictable outcome. This paper describes the therapeutic use of osseointegrated implants to replace congenitally missing upper lateral incisors. Highlighting the importance of the Orthodontic/Restorative interface.
\end{abstract}

\section{Introduction}

The successful use of dental implants to replace missing teeth has been one of the most popular, exciting and evolving areas of clinical dentist. When implants are thought as a treatment option, treatment planning has become more complex for the dental practitioner, and an interdisciplinary team approach is recommended $[1,2]$. Interdisciplinary approach would involve a preprosthetic and orthodontic treatment and following consultations with an oral surgeon and a restorative dentist, implant treatment was selected as a treatment modality [2-5].

The maxillary lateral incisor is the second most frequently missing tooth after the mandibular second premolar even though Muller et al. found that maxillary lateral incisors experience the most agenesis (not including third molars). Agenesis of the maxillary lateral incisor is also linked with anomalies and syndromes such as agenesis of other permanent teeth, microdontia of maxillary lateral incisors (peg laterals), palatally displaced canines and distal angulations of mandibular second Premolars [6,7] .

Absence of any tooth can cause treatment difficulties, but agenesis of the maxillary lateral incisor poses a unique set of restorative challenges. Because the maxillary lateral incisor is located in the esthetic zone, it is essential that bone height, papilla height, enamel color, and shape match the surrounding teeth. Clinicians attempt to maintain the proper anterior overbite; over jet and ideal inter-arch relationships of the canine teeth while creating enough space for a fixed partial denture or more commonly, an implant with a single crown restoration, but few treatment options are available for patients with agenesis of one or both maxillary lateral incisors. One option is to close the space(s) and restore the remaining teeth accordingly and the second is to open the space for a fixed partial denture or implant [7-10] (Figure 1).

Since the development of genetic mapping, Brook et al. have shown that some genes are implicated in the agenesis of teeth, including PAX9, MSX1 and AXIN2. The PAX9 gene is on chromosome 14 with a controlling factor for dental development and mutations related to missing teeth [11]. Brook et al. measured the tooth sizes on maxillary and mandibular dental casts in the test group, 10 people with a known PAX9 mutation in one family and 10 people in a control group matched for sex, age and ethnicity, who were not related to the test group and did not have the PAX9 mutation. Differences in the test group with the mutation and hypodontia were found; these teeth were significantly smaller than controls. Canines and first molars were least affected in the test group [11]. This contradicts Bailit's theory that genetics mostly affects the first tooth in each group: the central incisor, canine, first premolar and molar [12]. Brook, et al. found that the second tooth in each group was more affected by the PAX9 mutation. The study concluded that the PAX9 mutation not only decreased tooth number, but also tooth size throughout the dentition [11].

Peck and Kataja linked palatally displaced canines, transposition of mandibular lateral incisors and canines and maxillary canine and premolar transposition with agenesis [13]. They studied 161 subjects and found that patients with maxillary canine-first premolar transposition were 13 times more likely $(26 \%)$ to have agenesis of a maxillary lateral incisor. They agreed with Brook et al. that PAX9 and MSX1 mutations contribute to tooth agenesis20, however they also pointed out that the PAX9 and MSX1 genetic mutations are associated with posterior tooth agenesis while a strong causative gene mutation for anterior agenesis has yet to be found. Peck, Peck and Kataja believe signaling proteins such as bone morphogenic proteins (BMP) and

Correspondence to: Abu-Hussein Muhamad, DDS, MScD, MSc, M Dent Sci (Paed Dent), FICD 123 Argus Street 10441 Athens, Greece, E-mail: abuhusseinmuhamad@gmail.com

Key words: congenitally missing lateral incisor, interdisciplinary treatment, dental implant

Received: July 25, 2016; Accepted: August 08, 2016; Published: August 11, 2016 


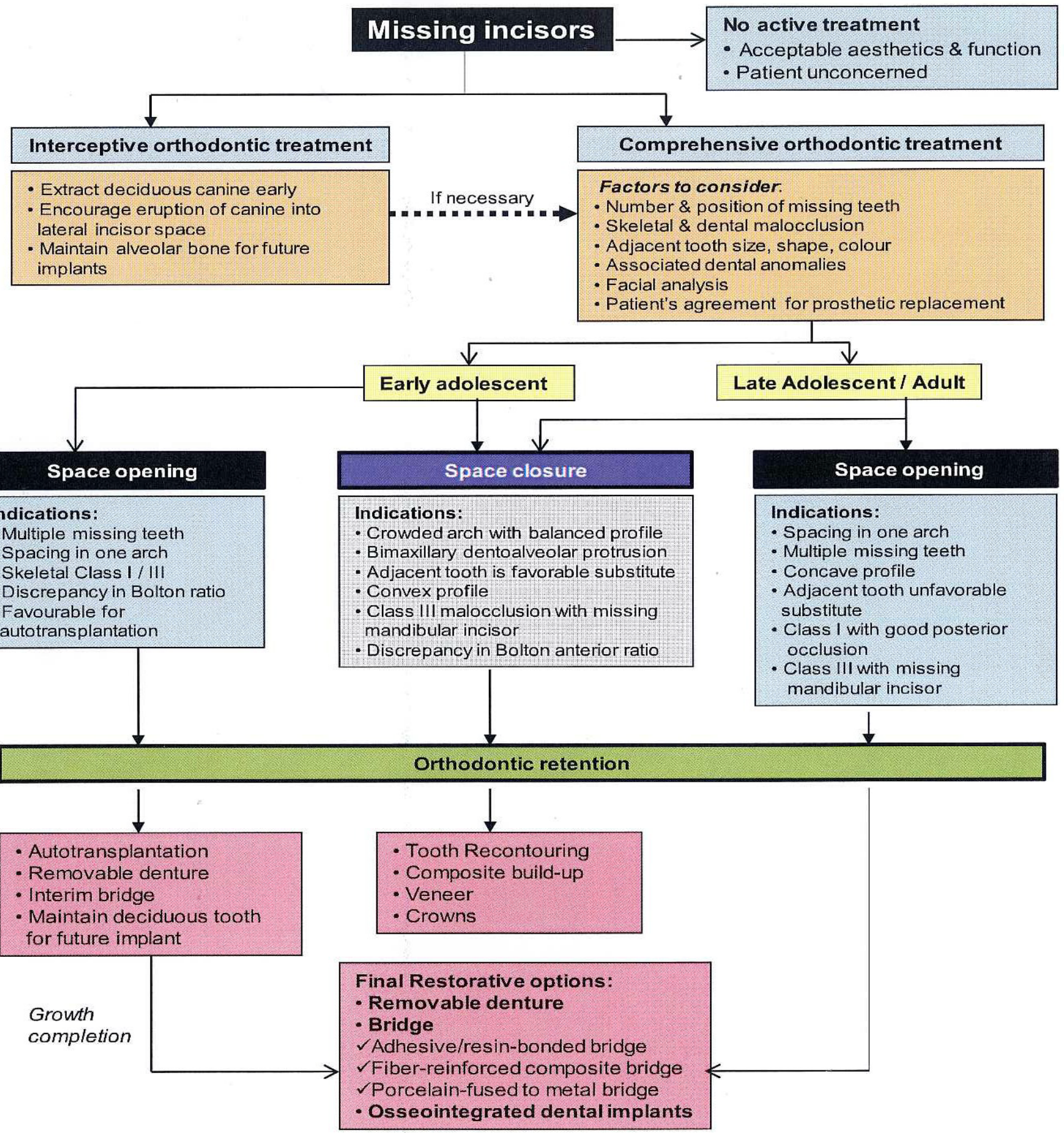

Figure 1. Algorithm of "Orthodontic management of developmentally missing incisors".

fibroblast growth factor (FGF) may be responsible for agenesis early in embryonic development [13].

Arte, et al. mapped the genes of 77 individuals in 3 generations of Finnish families to determine which genes affect incisor-premolar hypodontia. Thirty-one of the 77 participants expressed incisorpremolar hypodontia; however children under the age of 6 were excluded from the study because hypodontia cannot reliably be ascertained at that age. They hypothesized that epidermal growth factor (EGF), epidermal growth factor receptor (EGFR), and fibroblast growth factor (FGF-3) genes were responsible for incisor-premolar hypodontia because: 1) EGF has been implicated in early tooth morphogenesis in mice, 2) EGFR is expressed in developing teeth from the bud stage onward, and 3) FGF-3 is intensely expressed in dental mesenchyme during cap and bell stages of tooth development. However, after extracting DNA from blood samples of the participants and running PCR procedures, results did not show that these genes are involved in incisor-premolar hypodontia.

They concluded that mutations in EGF, EGFR, FGF-3 and FGF-4 
did not influence incisor-premolar hypodontia in the families studied [14].

Genetic factors appear to play a large role in tooth size and agenesis with the PAX9 and MSX1 mutations; however some authors suspect that the local environment is important factor [7]. EGF, EGFR, FGF-3 and FGF-4 are not shown to be linked to incisor-premolar agenesis, but it is possible that signaling factors early in embryologic development may contribute to agenesis. Through the work of Pirinen, et al. and Arte et al., it is evident that incisorpremolar hypodontia is genetically inherited, with strong links to other dental anomalies such as palatally impacted canines. Incisor-premolar hypodontia is an autosomal dominant gene inheritance with incomplete penetrance $[14,15]$.

The incidence of congenitally missing maxillary lateral incisors has been reported to range from between $1 \%$ and $2 \% 1$ to as high as $5 \%$. Maxillary lateral incisors are the most common congenitally missing teeth after upper and lower second premolars. Sex differences have been found to be negligible, with slightly more females affected as compared with males $[16,17]$. Treatment options include orthodontic movement of cuspids into lateral incisor sites, prosthodontic restorations including fixed and removable prostheses and resin-bonded retainers, and single tooth implants [9]. Implants do not require preparation of natural teeth and thus can be considered the most conservative approach. Orthodontic space opening may be necessary but on occasion can compromise esthetics, periodontal health, and function $[1,2]$.

Parents and professionals must often decide at a child's early age on how to cope with congenitally missing maxillary lateral incisors. Orthodontic treatment to create space for implants should not be initiated before the age of 13 . This will avoid the potential for alveolar bone atrophy and the risk of relapse and subsequent retreatment $[3,4]$. The management of small restorative areas in the esthetic zone has posed significant problems for the implant and restorative team. The lack of bone available for the surgeon as well as the lack of restorative space available between the adjacent teeth makes tooth replacement with implants challenging for both the restorative dentist and the laboratory technician. In the past, patients with congenitally missing teeth or microdontia have been treated with resin-bonded bridges, removable retainers, or cantilever crowns to avoid the use of standard-diameter implants and prosthetics in this area $[1,3]$. The two common treatment options include orthodontic space opening for future restorations or orthodontic space closure utilizing the adjacent permanent canine teeth. With a paradigm shift in the patient expectations towards functional as well as esthetically appealing replacements for edentulism, the implant based oral rehabilitation has emerged as a sole winner in fulfilling all aspects of patient needs [1-5].

The orthodontist plays a key role in determining and establishing space requirements for patients with congenitally missing maxillary lateral incisors .However, the implant based treatment option in such patients requires an interactive and interdisciplinary management approach [1,3,5]. This interdisciplinary approach may involve preprosthetic orthodontic treatment following consultations with an oral surgeon or periodontics and restorative dentist to ensure orthodontic alignment will facilitate the surgical, implant and restorative treatment. Too often, surgeons attempting to place standard-diameter implants have forced the restorative team to manage these small dimensions with a lack of adequate prosthetics because of the size and diameter of the fixture head. Recently, manufacturers in the implant industry have offered a 3-mm diameter implant design to address these challenges $[3,4,5]$. Most of the implants available in the 3-mm size have been one-piece or unibody implants, which often necessitate conventional tooth-preparation techniques by the restorative team as well as standard cord-impression techniques for indexing the restorative margins. With some systems, there is no need for preparation due to a cervical marginal collar that can be captured utilizing a snap-in impression transfer [16-20].

This paper describes the therapeutic use of osseointegrated implants to replace congenitally missing upper lateral incisors. Highlighting the importance of the Orthodontic/Restorative interface.

\section{Case report}

The initial clinical exam revealed diastema, congenitally missing maxillary lateral incisors with the canines located in the lateral incisor positions, and the primary maxillary canines still located in their original positions. These aspects created not only esthetics deficiencies but also maloclussion. Therefore, a multidisciplinary treatment was suggested to restore both esthetics and function [9].

\section{Phase 1: Planning}

All dental professionals involved in the treatment (orthodontist, periodontics, master ceramist, and operative dentist) evaluated the clinical case individually to decide which noninvasive procedures were indicated. Next, the four professionals discussed the prognosis and limitations of the case. The master ceramist performed a diagnostic wax-up to provide a model of the multidisciplinary treatment. After patient approval, the conservative treatment was then split into three restorative phase orthodontic, surgical, and restorative.

\section{Phase 2: Orthodontics}

Dental implants have become a common method for restoring missing teeth. However, especially upper lateral incisor implants are esthetically challenging. The orthodontic improvement of the procedure and the final attendance result of these patients can be accomplished best by positioning the remaining natural dentition in the anatomically correct location. This treatment should be closely coordinated with the implant placement and the restorative team. In cases of extensive dento-alveolar and skeletal malformations, occlusion and facial proportions additionally must be improved by orthogenetic surgery and sometimes even by esthetic plastic surgery.

The orthodontic treatment used the following parameters for evaluation: sagittal relationship between the dental arches; posterior occlusion; location, shape, and size of the canines; amount of remaining interdental space; and profile and facial skeletal pattern of the patient.

After orthodontic treatment was finalized, the orthodontic brackets were removed and a removable appliance was used to replace the missing maxillary lateral incisors (Figure 2).

\section{Phase 3: Surgical}

A more recent option for treating congenitally missing lateral incisors, and one that currently is recommended often, is the singletooth implant. Over the past several years, the predictability and long term success rates of implants have made them an obvious restorative choice [5], especially when teeth adjacent to the space are healthy, of normal size and shape, and unrestored. Furthermore, placement of an implant may provide a functional stimulus to help preserve bone and prevent resorption. However, when choosing the single-tooth implant as a restorative option, several factors must be taken into account such as growth considerations, space requirements, and site development $[2,3,15]$. 


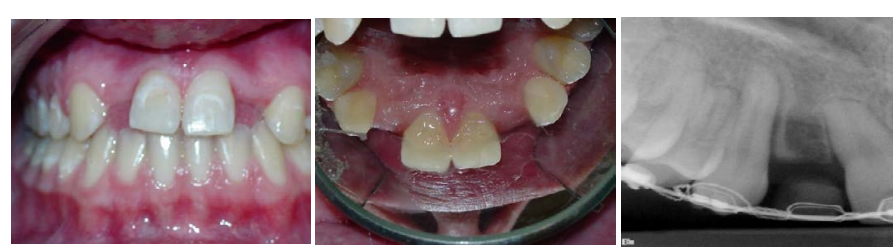

Figure 2. a. Immediately post-orthodontic treatment, b. Adequate keratinized tissue present Bone sounding revealed adequate width and c. inadequate mesial to distal width $\# 12$.

Because an implant acts essentially like an ankylosed tooth, any vertical alveolar growth and eruption of teeth would cause a discrepancy between the gingival margin of the natural tooth and the implant. Therefore, implant placement should occur only after growth has been completed, and it has been suggested that neither chronological age nor hand-wrist radiographs are reliable enough to make that determination. Instead it would be best to compare superimposed cephalometric radiographs taken at 1-year intervals until no growth changes are detected [16,17]. Also, the amount of space between the roots is critical to successful implant placement, and orthodontic intervention usually is necessary to achieve not only the amount of interradicular space needed, but also the proper rootarigulation. Because orthodontic treatment usually occurs at an early age, several years of maintenance therapy may be required until the appropriate age for implant placement. It is also important to maintain proper spacing for ideal tooth proportions of the final restoration In addition to the tooth width requirements for mesiodistal spacing, the alveolar width in a buccolingual direction must be adequate for implant placement. Often an additional surgical appointment is necessary to graft or augment the alveolar ridge before an implant can be placed. It has been suggested in the literature that by allowing or guiding the eruption of the canines into the lateral position and orthodontically moving them to their natural position, the necessary amount of buccolingual alveolar thickness for implant placement can be achieved naturally, without the need to perform any ridge augmentation $[17,18]$.

Although not completely understood, it has been shown that very little, if any, resorptive change in alveolar bone width is observed when space is opened orthodontically compared with the decrease in alveolar ridge width after extraction of maxillary anterior teeth. However, a disadvantage of orthodontic canine distalization for implant site development is the potential for loss of arch length when the canines are allowed to eruptmesially $[9,17,18,19]$.

When agenesis of maxillary lateral incisors is diagnosed in a young patient, usually primary maxillary lateral incisors are retained. In such cases, it may be necessary to selectively extract the primary lateral incisors to encourage the permanent canine to erupt mesially, adjacent to the central incisor.

The canine will influence the thickness of the edentulous alveolar ridge due to its large buccolingual width; otherwise the osseous ridge will not fully develop due to the absence of the lateral incisor $[2,4,18]$.

As the canine is moved distally to open space for the lateral incisor implant and crown, the root movement creates an increased and adequate alveolar ridge which allows proper implant placement. However, the time of implant placement should be relative close to the orthodontic treatment. This procedure is called "Implant site development". If inadequate alveolar ridge is present, ridge augmentation may be necessary using bone grafts [19].

Adequate implant space: The amount of space needed for the implant and crown is generally determined by the contralateral lateral incisor. However, if both lateral incisors are missing or the contralateral one is peg-shaped, the amount of space should be determined by one of the methods below:

\section{$>$ The golden proportion or a recurrent esthetic proportion \\ $>\quad$ The Bolton analysis \\ $>$ A diagnostic wax-up \\ $>\quad$ Mean values}

The small size of the maxillary lateral from 5, 5-8, $0 \mathrm{~mm}$ requires careful planning for an implant to be placed. It's important that orthodontic movement has distanced not only the crowns, but the roots of the adjacent teeth too. Generally, the adequate coronal space should be no less than $6,3 \mathrm{~mm}$ whereas the interradicular space no less than $5.7 \mathrm{~mm}$. «At least, 1, $5 \mathrm{~mm}$ between of the implant and adjacent roots is desirable as it is cited that narrower distances between them are more likely to show a reduction in bone height over time. In addition, fixed retention is suggested rather than removable appliances to prevent relapse crowns, but the roots of the adjacent teeth too $[2,9,10,11]$. Generally, the adequate coronal space should be no less than $6,3 \mathrm{~mm}$ whereas the interradicular space no less than $5.7 \mathrm{~mm}$. «At least, $1,5 \mathrm{~mm}$ between of the implant and adjacent roots is desirable as it is cited that narrower distances between them are more likely to show a reduction in bone height over time. In addition, fixed retention is suggested rather than removable appliances to prevent relapse.

Generally, implants must not be placed until the patients have completed their facial growth and the majority of their tooth eruption $[2,6,8,11]$. As the face grows and the mandibular rami lengthen, teeth must erupt to remain in occlusion. However, the implant behaves like an ankylosed tooth and will not follow the changes of the alveolar processes due to the eruption of adjacent teeth. This may result in clinical infra occlusion of the implant supported crown and cause a discrepancy in the occlusal plane and between the gingival margins of the implant and the adjacent natural teeth. Thus, evaluation of the completion of facial growth by cephalometric radiographs must be done and subsequently, the patient should be informed for the optimal time of implant placement. However, even mature adults can exhibit major vertical steps after anterior restorations with implants to the same extend as adolescents (Figures 3 and 4 ).

\section{Phase 4: Restorative}

Six weeks after surgery the patient returned for the restorative phase of treatment. The healing abutment on the implant was then modified to create a better emergence profile $(1,2, \%)$. This was achieved with air abrasion of the healing abutment, application of metal primer, bonding agent and flowable composite. The desired effect was achieved in that the soft tissue moved in a bucco-apical direction creating a more labial emergence profile. A harmonious gingival contour with the adjacent teeth was established. It was suggested from the outset that a crown lengthening procedure on the peg shaped lateral would create a longer crown length and a more symmetrical gingival contour in relation to the contra-lateral incisor $[4,7,8,11]$. The patient decided to keep treatment simple and avoid further surgery and cost [2].

An open tray $\mathrm{NC}$ impression coping was connected to the implant and verified radiographically. The 12,22 was minimally prepared for a full coverage veneer. A polyether impression compound was used to take the final impression, taking great care to record the soft tissue emergence profile. 

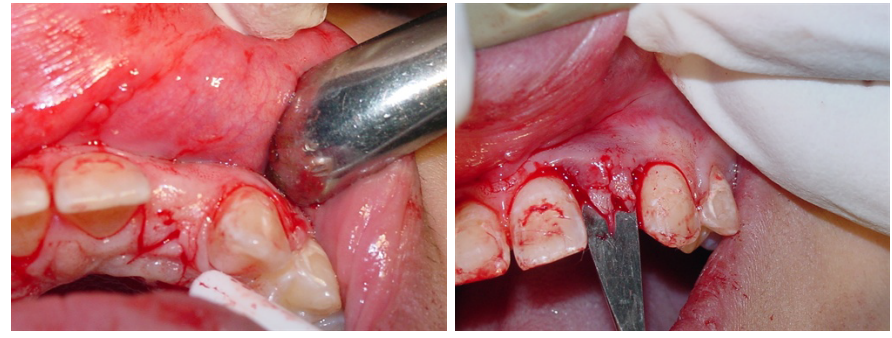

Figure 3. a. Instead of a midcrestal incision, a modified incision was used. Midcrestal incisions tend to produce an "envelope effect" when approximating tissue around an abutment and $b$. The fingers are visible.
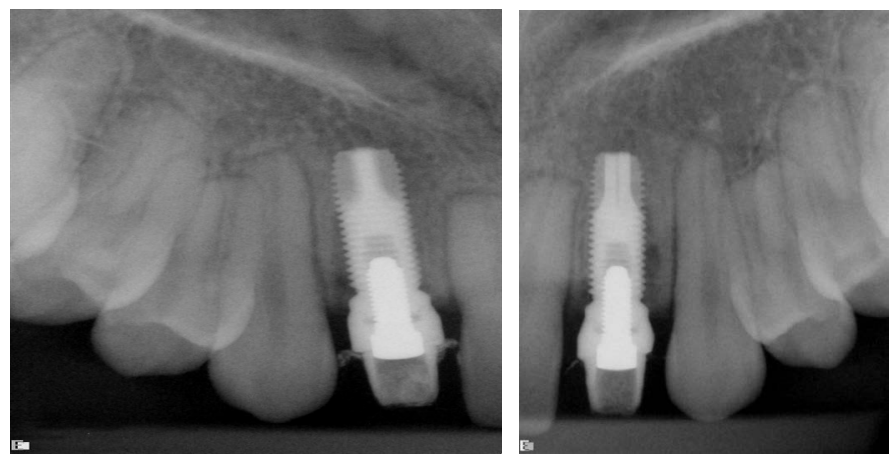

Figure 4. a. 3I $3.75 \times 13 \mathrm{~mm}$ placed to level of crest. The platform has a bevel that rests on the cortical bone but is not countersunk. The fixtures were approximately at $50 \mathrm{Ncm}$ as the motor indicated and b. 3I $3.75 \times 13 \mathrm{~mm}$ placed to level of crest. The platform has a bevel that rests on the cortical bone but is not countersunk. The fixtures were approximately at 50 $\mathrm{Ncm}$ as the motor indicated. The abutments were prepared mostly extraoral and torqued to $32 \mathrm{Ncm}$. Acrylic temporaries fabricated and temporarily cemented.

A customised final abutment was cast accordingly and torqued to $35 \mathrm{Ncm}$. The porcelain fused to metal crown was cemented with Tempbond. The Emax full coverage veneer was luted with transparent Rely-X veneer cement, and the upper Hawley retainer adjusted to fit (Figure 5-7).

\section{Discussion}

For patients with congenitally missing lateral incisors, in addition to over-retained primary teeth, permanent canines may erupt or drift mesially into the edentulous space. If the space is to be opened orthodontically for ideal prosthesis, the canines will need to be moved distally, which may result in development of the alveolar ridge in the canine region $[2,3,5]$. In cases where the occlusion and esthetics of the canine in the lateral position are acceptable, closure of the lateral space by the mesially positioned canine may be the simplest alternative treatment option. However, in all the above congenital missing cases we considered space opening followed by fixed prosthesis would be more acceptable on aesthetic point of view [19,20,21].

Esthetics as well as occlusion must be considered in the final orthodontic positioning of the teeth adjacent to the edentulous space. To satisfy the "golden proportion" principle of esthetics, the space for the maxillary lateral incisor should be approximately two-thirds of the width of the central incisor.

However, if the patient is missing only one maxillary lateral incisor, the space required to achieve symmetrical esthetics and occlusion is primarily dictated by the width of the contralateral incisor [22].

The optimal time for placement of fixed prosthesis is after the growth of the maxilla, mandible and alveolus is complete. If fixed prosthesis/implant are placed before growth is complete, the surrounding alveolar bone may continue to develop vertically and adjacent teeth may continue to erupt. Thus a discrepancy between the gingival margins of the prosthesis and the natural teeth is created and the prosthesis appears to became submerged. This creates a functional as well as an esthetic problem [21-25].

The correction of this aesthetic problem can be a diagnostic and clinical challenge in dental practice $[8,9]$.

\section{Treatment options include [22]}

No treatment/simple improvement with composite resin: Patient's with minimal spacing may feel that the appearance of their teeth is satisfactory. For others, composite resin can be used to improve tooth appearance by closing a small diastema.

Space Closure: The space corresponding to missing lateral incisors may be closed by protraction of the cuspids and the buccal segments with the final objective being a class II buccal occlusion. The cuspids and first bicuspids can be masked to simulate the lateral incisor and cuspid, respectively using composite resin.

Space Opening: The alternative to space closure is to maintain or create the necessary space, for a prosthetic replacement of the missing tooth. Replacement options have been a removable partial denture, conventional bridges, resin bonded bridges and single tooth implants $[2,3,5]$. Each one has its own indications, advantages and disadvantages.

Osseointegrated implants may be used to restore single unit spaces

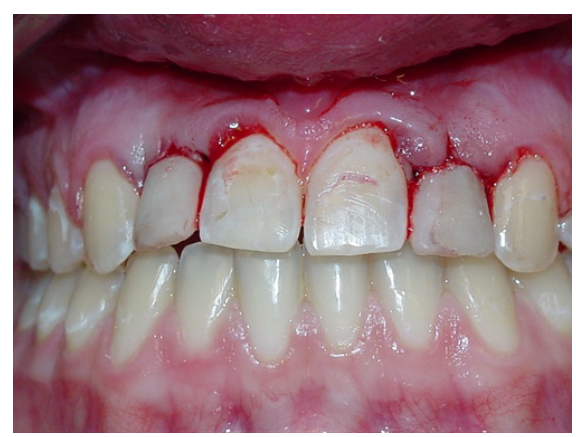

Figure 5. Immediately post operation.
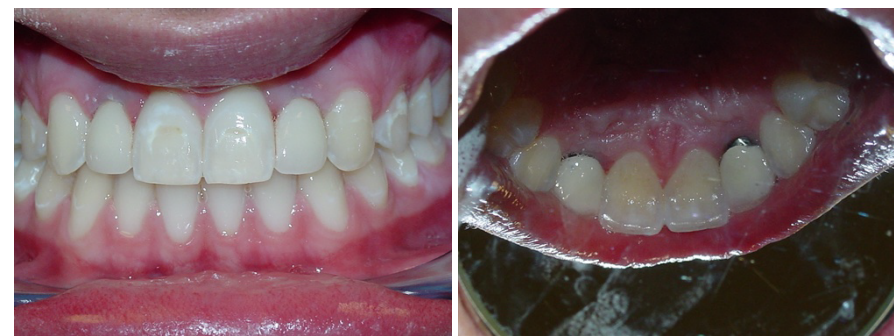

Figure 6. a. Immediately post insertion and b. Lingual view.
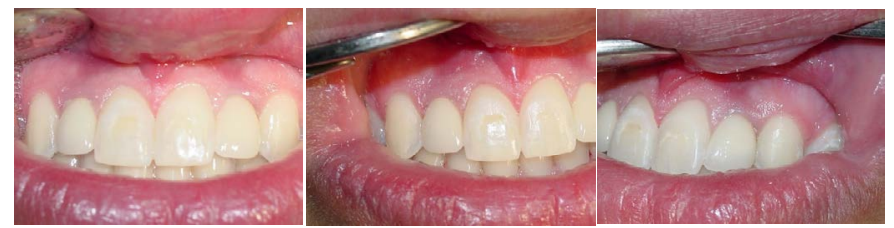

Figure 7. a. One year follow up, b. One year follow up. Tissue matured well\#12 and c. One year follow up. Tissue matured well\#22. 
including the congenitally missing maxillary lateral incisor. However, implants are not indicated in patients for whom a potential for further growth exists since an implant, which does not have periodontal ligament, cannot erupt and keep pace with dentoalveolar development [9]. Single tooth implants are likely to be of most use in the adult patients.

Patient selection should be considered carefully keeping in mind oral hygiene socio-economic background and ability of patient to follow maintenance instructions [23,24].

The importance of early diagnosis of missing teeth should not be forgotten, so that a multidisciplinary approach can be established at an early stage. Preparatory orthodontic treatment may be needed to align teeth, create adequate space in addition to establish an optimal axial inclination of the teeth adjacent to the proposed implant site. With proper patient selection and diagnosis, understanding of occlusion, effective communication among operators and attention to detail, the single tooth restoration can be a predictable esthetic and long-lasting restorations $[1,2,21,22,25]$.

In this case, alveolar bone was available in maxillary lateral incisor areas in the mesiodistal and coronoapical dimension; however, there was deficiency in orofacial dimension. The patient was refused to have bone augmentation procedures using either autogenic or synthetic bone grafts because of financial and patient related factors [19]. Therefore, implants with $3 \mathrm{~mm}$ diameter were used to compensate for horizontal alveolar bone deficiency. However, to avoid labial fenestration, the implants had to be placed off axis in labial direction. The relationship of the position between the implant and the proposed restoration should be based on the position of the implant shoulder, since it will influence the final hard and soft tissue response [20]. The malposition of the implant shoulder in the coronoapical direction causes soft tissue recession. In this case, location of the implant shoulders was in coronoapical and mesiodistal dimension in comfort zone. However, in the orofacial dimension the implant shoulders were in danger zone $[2,26,27]$. The angulation of implants in labial direction was compensated using angled abutments that were prepared for better emergence profile of the ceramic crowns. Many authors have also concluded that angled abutments may be considered a suitable restorative option when implants are not placed in ideal axial positions. Nevertheless, forces applied off axis may be expected to overload the bone surrounding single-tooth implants, as shown by Papavasiliou, et al. using finite element analysis. Hence, the segmental osteotomy may provide an alternative treatment to reposition the severely malposed implants [28].

\section{Conclusions}

Orthodontic space closure and implant substitution of missing maxillary incisors produced similar satisfactory esthetic results. Neither of the treatments impaired temporomandibular joint function.

However, orthodontic space closure patients had better periodontal health in comparison with implant substitution patients. Furthermore, infra occlusion more than $1 \mathrm{~mm}$ was noticed in all the implant patients.

\section{References}

1. Abu-Hussein M, Abdulgani A, Watted N, Zahalka M (2015) Congenitally Missing Lateral Incisor with Orthodontics, Bone Grafting and Single-Tooth Implant: A Case Report. J Dent Med Sci 14: 124-130.

2. Abdulgani A , Kontoes N , Chlorokostas G, Abu-Hussein M (2015) Interdisciplinary Management Of Maxillary Lateral Incisors Agenesis With Mini Implant Prostheses: A Case Report. J Dent Med Sci 14: 36-42.
3. Abusalih A, Ismail H, Abdulgani A, Chlorokostas G, Abu-Hussein M (2016) Interdisciplinary Management of Congenitally Agenesis Maxillary Lateral Incisors: Orthodontic/Prosthodontic Perspectives. J Dent Med Sci 15: 90-99.

4. Abu-Hussein M, Watted N, Abdulgani A, Borbély B (2015) Modern Treatment for Congenitally Missing Teeth: A MultidisciplinaryApproach. Int J Maxillofac Res 1 : 179-190.

5. Abu-Hussein M, Chlorokostas G, Watted N, Abdulgani A, Jabareen A (2016) PreProsthetic Orthodontic Implant for Management of Congenitally Unerupted Lateral Incisors - A Case Report. J Dent Med Sci 15: 99-104.

6. Muller TP, Hill IN, Peterson AC, Blayney JR (1970) A survey of congenitally missing permanent teeth. $J$ Am Dent Assoc 81: 101-107. [Crossref]

7. Abu-Hussein M, Watted N, Yehia M, Proff P, Iraqi F (2015) Clinical Genetic Basis of Tooth Agenesis. J Dent Med Sci 14: 68-77.

8. Abu-Hussein M, Watted N, Abdulgani A, Kontoes N (2015) Prosthodontic-Orthodontic Treatment Plan with Two-Unit Cantilevered Resin-Bonded Fixed Partial Dentur IOSR-JDMS 14: 131-136

9. Muhamad AH, Azzaldeen A, Nezar W, Mohammed Z ( 2015) Esthetic Evaluation of Implants Placed after Orthodontic Treatment in Patients with Congenitally Missing Lateral Incisors. J Adv Med Dent Sci Res 3: 110-118.

10. Abdulgani M, Abdulgani AZ, Abu-Hussein M (2016) Two Treatment Approaches for Missing Maxillary Lateral Incisors: A Case. J Dent Med Sci 15: 78-85.

11. Brook AH, Elcock C, Aggarwal M, Lath DL, Russell JM, et al. (2009) Tooth dimensions in hypodontia with a known PAX9 mutation. Arch Oral Biol 54 Suppl 1 S57-62. [Crossref]

12. Bailit HL (1975) Dental variation among populations. An anthropologic view. Dent Clin North Am 19: 125-139. [Crossref]

13. Peck S, Peck L, Kataja M (2002) Concomitant occurrence of canine malposition and tooth agenesis: evidence of orofacial genetic fields. Am J Orthod Dentofacial Orthop 122: 657-660. [Crossref]

14. Arte S, Nieminen P, Pirinen S, Thesleff I, Peltonen L (1996) Gene defect in hypodontia: exclusion of EGF, EGFR, and FGF-3 as candidate genes. $J$ Dent Res 75: 1346-1352. [Crossref]

15. Pirinen S, Arte S, Apajalahti S (1996) Palatal displacement of canine is genetic and related to congenital absence of teeth. J Dent Res 75: 1742-1746. [Crossref]

16. Abu-Hussein M, Watted N, Watted A, Abu-Hussein Y, Yehia M, et al. (2015) Prevalence of Tooth Agenesis in Orthodontic Patients at Arab Population in Israel. Int J Public Health Res 3: 77-82.

17. Abu-Hussein M, Watted N, Azzaldeen A, Yehia M, Awadi O, et al. (2015) Prevalence of Missing Lateral Incisor Agenesis in an Orthodontic Arabs Population in Israel (Arab48). Int J Public Health Res 3: 101-107.

18. Muhamad AH, Azzaldeen A (2012) Autotransplantation of Tooth in Children with Mixed Dentition. Dentistry 2: 149.

19. Abu-Hussein M, Watted N, Abdulgani M, Abdulgani AZ (2016) Tooth Autotransplantation; Clinical Concepts. J Dent Med Sci 15: 113.

20. Abu-Hussein M, Nezar W, Azzaldeen A, Abdulgani M (2016) Prevalence of Traumatic Dental Injury in Arab Israeli Community. J Dent Med Sci 15: 91-98.

21. Kokich VO Jr, Kinzer GA (2005) Managing congenitally missing lateral incisors. Part I: Canine substitution. J Esthet Restor Dent 17: 5-10. [Crossref]

22. Savarrio L, McIntyre GT (2005) To open or to close space--that is the missing lateral incisor question. Dent Update 32: 16-18, 20-2, 24-5. [Crossref]

23. Rosa M, Zachrisson BU (2010) The space-closure alternative for missing maxillary lateral incisors: an update. J Clin Orthod 44: 540-549. [Crossref]

24. Kokich VG (2004) Maxillary lateral incisor implants: planning with the aid of orthodontics. J Oral Maxillofac Surg 62: 48-56. [Crossref]

25. Kinzer GA, Kokich VO Jr (2005) Managing congenitally missing lateral incisors. Part III: single-tooth implants. $J$ Esthet Restor Dent 17: 202-210. [Crossref]

26. Antonarakis GS, Prevezanos P, Gavric J, Christou P (2014) Agenesis of maxillary lateral incisor and tooth replacement: cost-effectiveness of different treatment alternatives. Int J Prosthodont 27: 257-263. [Crossref] 
27. Balshi TJ (1993) Osseointegration and orthodontics: modern treatment for congenitally missing teeth. Int J Periodontics Restorative Dent 13: 494-505. [Crossref]
28. Papavasiliou G, Kamposiora P, Bayne SC, Felton DA (1997) 3D-FEA of osseointegration percentages and patterns on implant-bone interfacial stresses. $J$ Dent 25: 485-491. [Crossref]

Copyright: (C2016 Muhamad AH. This is an open-access article distributed under the terms of the Creative Commons Attribution License, which permits unrestricted use, distribution, and reproduction in any medium, provided the original author and source are credited. 